\title{
Identification of Tigers for Census by the Method of Tiger Iris Pattern Matching and Recognition
}

\author{
Dibyendu Ghoshal \\ $\mathrm{PhD}$, Associate professor \\ Department of ECE \\ NIT Agartala
}

\author{
Parthasarathi De \\ Lecturer \\ Department of CSE \\ ICFAI University Tripura
}

\author{
Bapi Saha \\ Lecturer \\ Department of CSE \\ ICFAI University, Tripura
}

\begin{abstract}
The Iris pattern of any animal (including human being) is statistically unique and suitable for biometric measurements. The identity of the animal concerned can be determined and verified comparing the templates obtained with the present algorithm with that template stored in database which was formed on the basis of previous studies. In the present study, the method of circular Hough transform is used for segmentation of the tiger Iris and subsequently Daugman's rubber Sheet model is used for normalization of the segmented values. Pattern matching is achieved by calculating Hamming Distance where its degree is proportional to the closeness of matching. The closer matching between the stored and calculated pattern is found to lead towards better recognition of Irises and thereby the animal itself.
\end{abstract}

\section{Keywords}

Iris recognition, Pattern matching, biometric identification.

\section{INTRODUCTION}

Biometric Impressions or attributes have been found to be unique for every individual as well as animals .Irises in the eye can serve as one of the biometric parameters which have been used for identification and authentication of the humans or animals. The features of the tiger Iris (like any other animal or bird) can be measured with the help of various algorithms and the Iris patterns thus found to be statistically unique [1-3].

Normally animals in the forest are counted based on their footprints or pugmarks which are also some kind of biometric impression. Wild Life conservations catalogue pugmarks and this has been found to be a tedious process. The Pugmarks are prone to be distorted or obliterated with time and weather variations and it leads to immense problem during annual census [4].On the contrary, the images of the tiger Irises can be acquired with the help of static camera to be located at various places within the forests and the Iris images can be analyzed for the matching and recognition of the same which are already stored in the database of the Iris images collected from the same area. The entire process may be considered as one of the most reliable forms of biometric technology.

A number of studies already been reported since long on Iris recognition. The visible texture of human Iris in a real time video image has been encoded into a compact sequence of multiscale quadrature using two dimensional Gabor wavelet coefficient [5].An automated method for Iris recognition was proposed by Wildes et al [6] and the study was based on a pyramid laplacian in order to perform two dimensional band pass decomposition for representing Iris Images. Studies on calculation of the zero crossing of the wavelet transforms were made [3] over concentric circles of the Iris and satisfactory results were obtained.

Although these studies were made for the detection of the Irises ,no work on Tiger Iris Recognition using combination of various algorithm is found in available published or on time literature. The tiger is a prominent animal in ecological system and hence its census is a point of interest for the wild life conservationist. In the present Study, the method of recognition of Tiger Iris is based on Circular Hough Transformation [4] for the purpose of the image segmentation. The extracted region has been normalized into a rectangular block with constant dimensions to account for imaging inconsistencies and then one dimensional Gabor Filter [2-3] is used to extract the phase data and quantize into four levels to encode the unique pattern of the Iris into a bit wise biometric template [6-7].

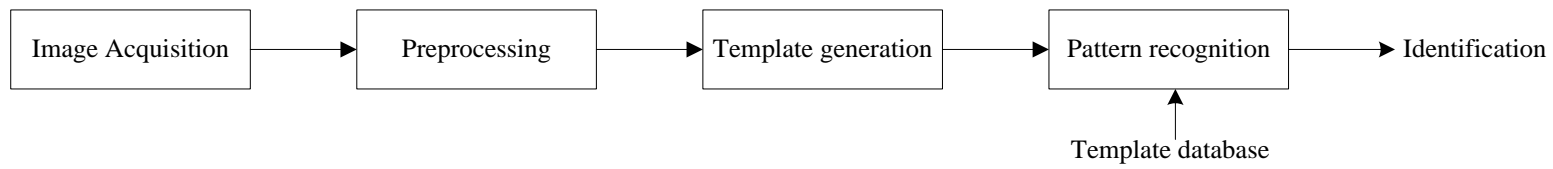

Fig 1: Block diagram of Tiger Iris Recognition System 


\section{TIGER IRIS RECOGINITION PROCESS}

\subsection{Image Acquisition}

First of all different images of Tigers eye of any particular tiger are taken from different angles from various stationary fixed points cameras in a particular area of any forest. Among these the best eye image is selected for iris segmentation for further processing which would lead to iris recognition in a meaningful way [4].

\subsection{Preprocessing}

An iris image contains some irrelevant parts such as eyelids sclera and pupil. Also, the size of an iris may vary depending on camera-to-eye distance, illumination level and amount of reflections. Therefore, the original image needs to be normalized. The process of normalization has been stated in subsection 2.2 .2 below.

\subsubsection{Iris Localization}

In Iris localization step the inner (iris/pupil) boundary and the outer (iris/sclera) boundary in the original image of the tiger eye are detected and are modeled as circles as shown in Fig 2.

\subsubsection{Iris Normalization}

Next step is to normalize iris images to compensate for iris deformation as shown in fig 3. The images of the Iris are required to be normalized to account for iris deformation and this has been shown in Fig 3.The iris region are transformed to have the equal dimensions. This will give fixed dimension of the irises and thus any comparisons between two photographs taken under different conditions will be possible. As such characteristic feature at different conditions can be compared.

Daugmans rubber sheet model has been used in the present study for the normalization of the isolated area of the image [1] and [6]. The central value of the pupil has been taken as the reference point, and the radial vectors are made to pass through that region. A normalized pattern is created through backtracking for finding the Cartesian coordinates of the relevant data points from the radial and angular positions in the normalized image pattern. The normalization process yields a two dimensional array with horizontal dimensions of angular resolution. The vertical dimensions of radial resolutions, in this way form the circular shaped correlate region. To avoid non iris region data from contaminating the normalized representations, the data points which are found to occur along the pupil border or the iris border are excluded [3, 9].

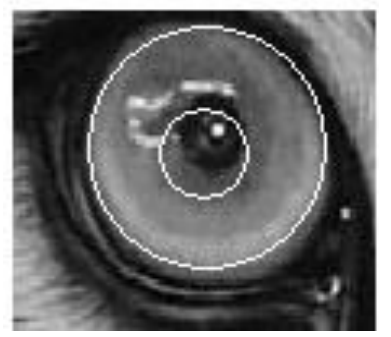

Fig 2: Iris Localization in which two circles overlay for iris and pupil boundaries.

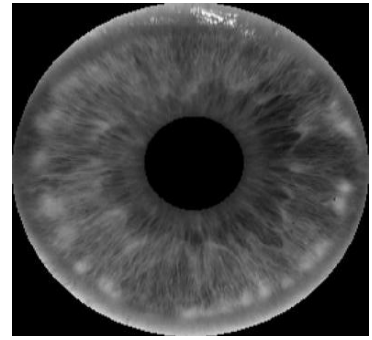

Fig 3: Segmented and normalized image of the tiger eye

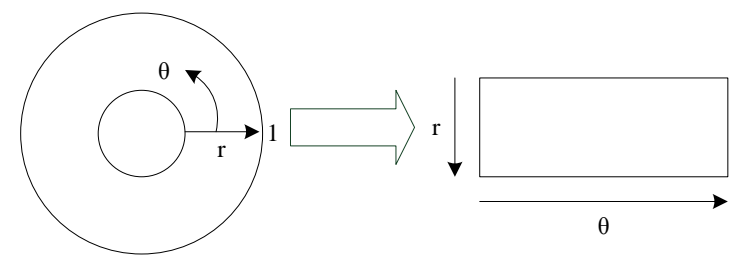

Fig 4: Schematic presentation of the unwrapping of the Iris.

The homogenous rubber sheet model devised by Daugman remaps each point within the iris region to a pair of polar coordinates $(\mathrm{r}, \theta)$ where $r$ is on the interval $[0,1]$ and $\boldsymbol{\theta}$ is angle $[0,2 \pi]$ which is shown in Fig4.

The remapping of the iris region from $(\mathrm{x}, \mathrm{y})$ Cartesian coordinates to the normalized non-concentric polar representation is modeled as [2-3]

$$
\begin{gathered}
I(x(r, \theta), y(r, \theta)) \rightarrow I(r, \theta) \\
\text { where } x(r, \theta)=(1-r) x_{p}(\theta)+r x_{I}(\theta) \\
\& y(r, \theta)=(1-r) y_{p}(\theta)+r y_{I}(\theta)
\end{gathered}
$$

Where $\mathrm{I}(\mathrm{x}, \mathrm{y})$ is the iris region image, $(\mathrm{x}, \mathrm{y})$ are the original Cartesian coordinates, $(\mathrm{r}, \theta)$ are the corresponding normalized polar coordinates, and $\mathrm{x}_{\mathrm{P}}, \mathrm{y}_{\mathrm{P}}$ are the coordinates of the pupil and $\mathrm{x}_{\mathrm{i}}, \mathrm{y}_{\mathrm{i}}$ are the coordinates of the iris boundaries along the $\theta$ direction. The rubber sheet model takes into account pupil dilation and size inconsistencies in order to produce a normalized representation with constant dimensions. In this way the iris region is modeled as a flexible rubber sheet anchored at the iris boundary with the pupil center as the reference point. This is shown in Fig 5[2, 6]. 


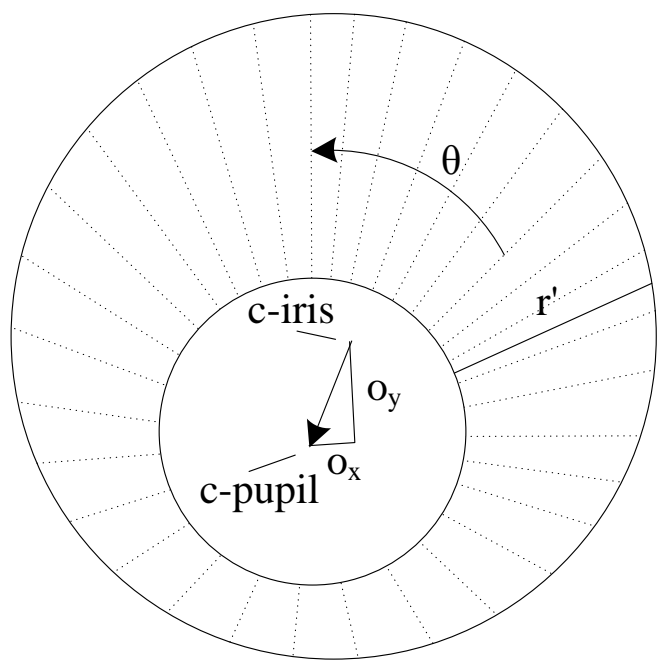

Fig 5: Schematic presentation of the variation of the pupil with respect to the Iris.

Along each and every radial line a number of data points are selected and this is defined as radial resolution. Around the Iris region there are number of radial lines which are circular in nature and is defined as angular resolution. And as the pupil cannot be always concentric to the iris a remapping formula is required to rescale points which will depend on the circle always $[2,6]$.

$r^{\prime}=\sqrt{\alpha \beta} \pm \sqrt{\alpha \beta^{2}-\alpha-r_{1}^{2}}$

$$
\alpha=o_{x}^{2}+o_{y}^{2}
$$

$\beta=\cos \left(\pi-\arctan \left(\frac{o_{y}}{o_{x}}\right)-\theta\right)$

Where the displacement of the pupil center relative to the Iris center is given by two parameters $o_{x}$ and $o_{y}$ and $r$ is the distance between the pupil edge and edge of the Iris at an angle $\theta$ and $r_{1}$ is the radius of the Iris.

The remapping formula first gives the radius of the iris region 'doughnut' as a function of the angle $\theta[6]$.

A fixed number of points are selected along each radial line irrespective of how narrow or wide the radius is at a particular angle. From the 'doughnut' iris region, normalization gives a two dimensional array with horizontal dimensions of angular resolution and vertical dimensions of radial resolution. Another two dimensional array was created for marking reflections, eyelashes, and eyelids found out in the segmentation stage. To prevent non-iris region data from corrupting the normalized representation, data points, which occur along the pupil border or the iris border, are discarded. [2] and [9].

\subsection{Feature Extraction and encoding}

Only the significant features of the iris must be encoded so that comparisons between templates can be made easier. The feature representation should have ample information to classify various irises and be less sensitive to noises. The figure 7 shows how the correlate region of the Iris has been extracted $[12,14]$.

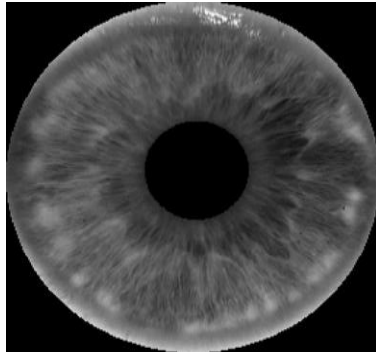

Fig 6: Segmented portion of the Iris

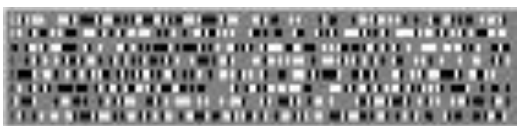

Fig 7: Correlate region of the Iris extracted from the segmented part of Iris.

In the correlate part of the iris some portion of it are white while rest of the portion is black. The black regions denotes the area for matching while the white regions of correlate denotes noise which is unwanted for our study given in fig below.

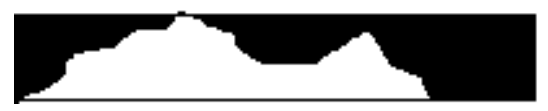

Fig 8: Black areas denotes area required for matching and White Region denotes the noise

\subsection{Encoding}

An easier way of using the Gabor filter is by breaking up the 2 Dimensional normalized pattern into a number of 1Dimensional wavelets, and then these signals are convolved with 1Dimensional Gabor wavelets. Gabor filters are actually used to extract localized frequency information. The frequency response of a Log-Gabor filter $[2,6]$ is given as

$$
G(f)=\exp \left(\frac{-\left(\log \left(f / f_{0}\right)\right)^{2}}{2\left(\log \left(\sigma / f_{0}\right)\right)^{2}}\right)^{2}
$$

Where $f$ represents the center frequency and $\sigma$ gives the bandwidth of the filter. 

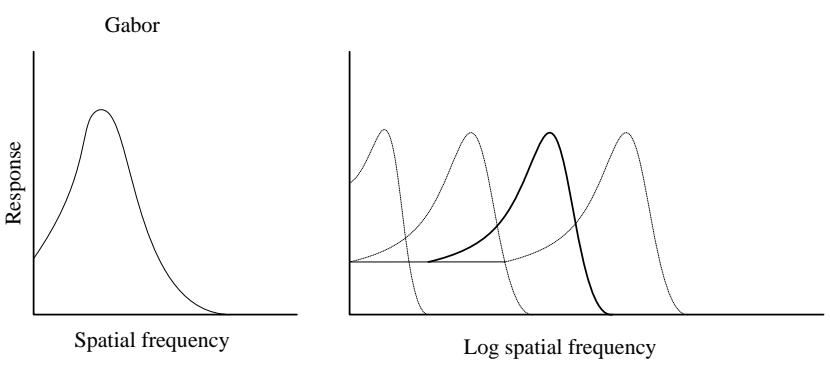

a
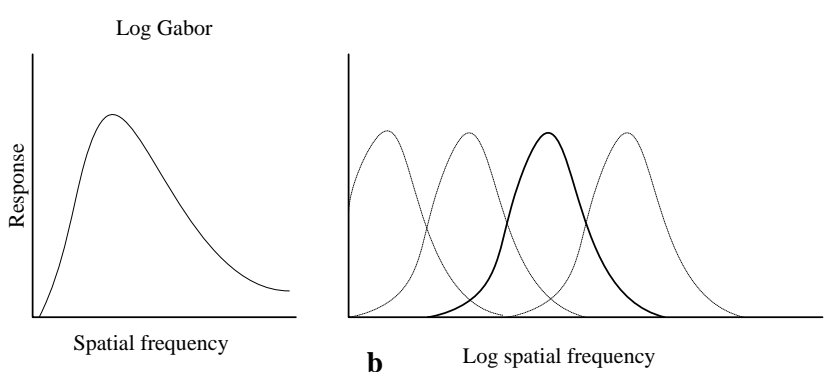

Fig 9 (a) Response of the Gabor filter in normal and logarithmic scale.(b) Response of the Log Gabor filter in normal and logarithmic scale.

\subsection{Matching}

Comparison of the bit patterns generated is done to check if the two irises belong to the same tigers. Calculation of Hamming Distance (HD) is done for this comparison. HD is a fractional measure of the number of bits disagreeing between two binary patterns [2]. Since this code comparison uses the iris code data and the noisy mask bits, the modified form of the HD is given by [1-2].

$$
H D=\frac{1}{N} \sum_{j=1}^{N} X_{j}(X O R) Y_{j}
$$

Where $\mathrm{Xj}$ and $\mathrm{Yj}$ are the two iris codes and $\mathrm{N}$ is the number of bits in each template. Since an individual iris region contains features with high degrees of freedom, each iris region will produce a bit-pattern which is independent to that produced by another iris, on the other hand, two iris codes produced from the same iris will be highly correlated.

If two bits patterns are completely independent, such as iris templates generated from different irises, the Hamming distance between the two patterns should equal 0.5 . This occurs because independence implies the two bit patterns will be totally random, so there is 50 percent probability of setting any bit to 1 , and vice versa. Therefore, half of the bits will agree and half will disagree between the two patterns. If two patterns are derived from the same iris, the Hamming distance between them will be close to 0 as they are highly correlated and the bits should agree between the two iris codes.

\section{CONCLUSION}

An area in which the algorithm is needed is to be continuously developed and improved had been outlined, and the potentials of utilizing Iris Recognition were examined.

Further biometric identification of tigers will be helpful to make a clear note of wild life preservation and also to make the census of tigers. Moreover differentiating of two species and also gender recognition will be possible in further studies.

\section{ACKNOWLEDGEMENT}

The author would like to thank .professor P.K .Bose, Director NIT Agartala, Dr Ajay Chakraborty for their constant support. I am also thankful to one of my senior lecturer Mr.Prakash Kumar of our Institute for his invaluable help.

\section{DEDICATION}

One of the author (D.Ghoshal) dedicates the study to the loveliest and everlasting memory of his only and younger sister Kumari Sumita Ghoshal who herself was a gem of scholars, a symbol of wisdom and wit, simplicity and dedication with peerless beauty in physique and mind.

\section{REFERENCES}

[1] J. Daugman, "How Iris Recognition Works," IEEE Trans. Circuits and Systems for Video Technology, vol. 14, no. 1, pp. 21-30,Jan. 2004.

[2] Libor Masek, "Recognition of Human Iris Patterns for Biometric Identification", School of Computer Science and Soft Engineering, the University of Western Australia, 2003.

[3] R. Wildes, J. Asmuth, G. Green, S. Hsu, R. Kolczynski, J. Matey, S. McBride. "A system for automated iris recognition. Proceedings IEEE Workshop on Applications of Computer Vision, Sarasota, FL, pp. 121128, 1994.

[4] Musgrave; Clyde (Frisco, TX),Cambier; James L. (Medford, NJ)." System and method of animal identification and animal transaction authorization using iris patterns". USPTO PATENT FULL TEXT AND IMAGE DATABASE Patent -6,424,727(23rd July, 2002).

[5] A.K. Jain, A. Ross, and S. Prabhaker, "An Introduction to Biometric Recognition," IEEE Trans. Circuits and Systems for Video Technology, vol. 14, no. 1, pp. 4-20, 2004.

[6] J. Daugman, "New Methods in Iris Recognition," IEEE Trans.System, Man, and Cybernetics-Part B: Cybernetics, vol. 37, no.5, pp. 1167-1175, 2007.

[7] R. Wildes. "Iris recognition: an emerging biometric technology". Proceedings of the IEEE, Vol. 85, No. 9 , 1997.

[8] Savita Sondhi, Sharda Vashisth, Asha Gaikwad and Anjali Garg. Article: IRIS Pattern "Recognition using Self-Organizing Neural Networks". IJCA Proceedings on National Conference on Advancement in Electronics and Telecommunication Engineering NCAETE (1):12-17.

[9] E.M. Arvacheh and H.R. Tizhoosh, "Iris segmentation: detecting pupil, limbus and eyelids," Proc.Int.Conf.Image Proc., 2453-2456, 2006.

[10] Abhyankara, A., Schuckers, S: "A novel biorthogonal wavelet network system for off-angle iris recognition", Pattern Recognition., 2010, 43, pp. 987-1007.

[11] Satyanarayana V V Tallapragada and E G Rajan. Article: IRIS Recognition based on "Non Linear Dimensionality Reduction of IRIS Code with KPCA and SVM based Classification". International Journal of Computer Applications 44(13):42-46, April 2012. 
[12] C. Boyce, A. Ross, M. Monaco, L. Hornak, and X. Li, "Multispectral "Iris Analysis: A Preliminary Study," Proc. IEEE Conf.Computer Vision and Pattern Recognition Workshop Biometrics,pp. 51-59, June 2006.

[13] Y. Zhu, T. Tan, and Y. Wang, "Biometric personal identification based on iris patterns," inProc. IEEE Int. Conf. Pattern Recognition., 2000, pp. 2801-2804.

[14] L. Ma, T. Tan, Y. Wang, and D. Zhang, "Personal identification based on iris texture analysis," IEEE Trans. Pattern Anal. Mach.Intell., vol. 25, no. 12, pp. 1519-1533,Dec. 2003.

[15] Gonzalez, R.C., Woods, R.E, Digital Image Processing, 2nd ed., Prentice Hall.

[16] Ya-Ping Huang, Si-Wei Luo, En- Yi Chen, "An efficient iris recognition system", International Conference on Machine Learning and Cybernetics, pp. 450-454, 2002.
[17] Mira J. and Mayer J., "Image feature extraction for application of biometric identification of iris: a morphological approach", IEEE Proc. XVI Brazilian Symposium on Computer Graphics and Image Processing, pp. 391- 398, 2003.

[18] Wood, N.M. Orlans, and P.T. Higgins, Biometrics, The McGraw-hill company, Berkeley, California, 2002.

[19] Z. Sun, T. Tan, and X. Qiu, "Graph matching iris image blocks with local binary pattern," In Proceedings of the International Conference on Advances onBiometrics (ICB '06), vol. 3832 of Lecture Notes in Computer Science, pp. 366-372, Springer, Hong Kong, January 2006.

[20] Zhu, Y., Tan, T., Wang, Y. 'Biometric personal identification based on iris patterns'. Proc. 15th Int Conf. on Pattern Recognition (ICPR), Spain, 2000, vol 2 ,

p.

2801 